\title{
还原氧化石墨烯修饰泡沫镍原位负载 $\mathrm{MnO}_{2}$ 对 $\mathrm{H}_{2} \mathrm{O}_{2}$ 电还原反应 催化性能的研究
}

\author{
宋聪颖孙逊叶克朱凯程鬼 \\ 间俊曹殿学王贵领* \\ (哈尔滨工程大学 材料科学与化学工程学院 哈尔滨 150001)
}

\begin{abstract}
摘要 采用两步易操作的水热法制备了还原氧化石墨烯 $(\mathrm{rGO})$ 修饰泡沫镍 $\left(\mathrm{Ni}\right.$ foam)基体原位负载 $\mathrm{MnO}_{2}$ 纳米片 $\left(\mathrm{MnO}_{2} / \mathrm{rGO} @ \mathrm{Ni}\right.$ foam) 催化剂电极. 通过 $\mathrm{X}$ 射线衍射 $\mathrm{XRD}$ )、扫描电子显微镜( $\mathrm{SEM}$ )、透射电子显微镜( TEM)对电极的 微观形貌和组成进行了表征. 利用循环伏安法和计时电流法对电极对 $\mathrm{H}_{2} \mathrm{O}_{2}$ 电还原反应的催化性能进行了系统的测试. 根据测试结果得知, 电极在 $3 \mathrm{~mol} / \mathrm{L} \mathrm{NaOH}$ 和 $1 \mathrm{~mol} / \mathrm{L} \mathrm{H}_{2} \mathrm{O}_{2}$ 溶液中表现出最佳的催化性能. 在该溶液中, 当电位为 -0.8 $\mathrm{V}$ 时, $\mathrm{H}_{2} \mathrm{O}_{2}$ 电还原反应的电流密度可以达到 $240 \mathrm{~mA} / \mathrm{cm}^{2}$, 高于同等条件下 $\mathrm{MnO}_{2}$ 直接生长在 $\mathrm{Ni}$ foam 上的电流密度 180 $\mathrm{mA} / \mathrm{cm}^{2}$. 通过不同温度下的极化曲线计算出了在该电极上 $\mathrm{H}_{2} \mathrm{O}_{2}$ 电还原反应所需的活化能大小为 $21.53 \mathrm{~kJ} / \mathrm{mol}$, 明显低 于文献中报道的数值. 对比实验结果表明 $\mathrm{rGO}$ 的加入显著地改善了 $\mathrm{MnO}_{2}$ 催化剂的催化性能与稳定性.
\end{abstract}

关键词 $\mathrm{MnO}_{2}$; 还原氧化石墨烯; $\mathrm{H}_{2} \mathrm{O}_{2}$; 电催化; 水热法

\section{Electrocatalytic Activity of $\mathrm{MnO}_{2}$ Supported on Reduced Graphene Oxide Modified Ni Foam for $\mathrm{H}_{2} \mathrm{O}_{2}$ Reduction}

\author{
Song, Congying Sun, Xun Ye, Ke Zhu, Kai Cheng, Kui \\ Yan, Jun Cao, Dianxue Wang, Guiling* \\ (College of Materials Science and Chemical Engineering, Harbin Engineering University, Harbin 150001)
}

\begin{abstract}
Fuel cells which use hydrogen peroxide as oxidant have been widely studied and presents good development foreground. As a liquid fuel, $\mathrm{H}_{2} \mathrm{O}_{2}$ possesses advantages of easily storage and transportation which make it can be widely used in underwater and space as a power source. At present, the most widely used catalysts for $\mathrm{H}_{2} \mathrm{O}_{2}$ electroreduction are noble metal catalysts. Compared with noble metals, transition metal oxides possess advantages of low cost and extensive sources. However, the catalytic activity of transition metal oxides is still much lower than noble metals. Therefore, many efforts should be made to improve the electrochemical performance of transition metal oxides. In this work, rGO is used as an additive to improve the electrochemcial performance of $\mathrm{MnO}_{2}$. An original electrode of $\mathrm{MnO}_{2}$ in-situ supported on reduced graphene oxide modified $\mathrm{Ni}$ foam $\left(\mathrm{MnO}_{2} / \mathrm{rGO} @ \mathrm{Ni}\right.$ foam) is prepared through two-step hydrothermal methods. Primarily, the novel current collector of $\mathrm{rGO} @ \mathrm{Ni}$ foam is obtained with larger surface area which is beneficial to the next loading of $\mathrm{MnO}_{2}$. Secondly, $\mathrm{MnO}_{2}$ is grown on the $\mathrm{rGO} @ \mathrm{Ni}$ foam also by a hydrothermal treatment. Besides large surface area, the addition of rGO can provide more channels for electron transfer and then accelerate the reaction rate of $\mathrm{H}_{2} \mathrm{O}_{2}$ reduction. The morphology and phase composition of the as-prepared electrode are investigated by measurements of X-ray diffractometer (XRD), scanning electron microscopy (SEM) and transmission electron microscope (TEM). It can be concluded from SEM and TEM images, both $\mathrm{rGO}$ and $\mathrm{MnO}_{2}$ exhibit sheet-like structure and there are many gaps existing between these sheets. Especially, the as-prepared $\mathrm{MnO}_{2}$ nanosheets builds a honeycomb structure which makes positive effects on the contact between $\mathrm{H}_{2} \mathrm{O}_{2}$ and catalyst. And XRD and HRTEM results show that $\mathrm{MnO}_{2}$ and $\mathrm{rGO}$ are successfully prepared on Ni foam. The electrochemical performance of the $\mathrm{MnO}_{2} / \mathrm{rGO} @ \mathrm{Ni}$ foam electrode toward $\mathrm{H}_{2} \mathrm{O}_{2}$ reduction is investigated by cyclic voltammetry and chronoamperometry in a three-electrode system in solutions of $\mathrm{NaOH}$ and $\mathrm{H}_{2} \mathrm{O}_{2}$. Results reveal that the reduction current density of $\mathrm{H}_{2} \mathrm{O}_{2}$ reduction on the $\mathrm{MnO}_{2} / \mathrm{rGO} @ \mathrm{Ni}$ foam electrode reaches $240 \mathrm{~mA} / \mathrm{cm}^{2}$ in a solution of $1.0 \mathrm{~mol} / \mathrm{L} \mathrm{H}_{2} \mathrm{O}_{2}$ and 3 $\mathrm{mol} / \mathrm{L} \mathrm{NaOH}$ at $-0.8 \mathrm{~V}$ which is much higher than that on $\mathrm{MnO}_{2}$ directly supported on $\mathrm{Ni}$ foam $\left(\mathrm{MnO}_{2} @ \mathrm{Ni}\right.$ foam). At the same time, a better stability is also achieved on the $\mathrm{MnO}_{2} / \mathrm{rGO} @ \mathrm{Ni}$ foam electrode. Generally speaking, the addition of rGO highly improves the electrocatalytic activity and stability of the as-prepared electrode indicating great application prospect in the future.

Keywords $\mathrm{MnO}_{2} ; \mathrm{rGO} ; \mathrm{H}_{2} \mathrm{O}_{2}$; electrocatalysis; hydrothermal method
\end{abstract}

* E-mail: wangguiling@hrbeu.edu.cn; Tel.: +86-451-82589036; Fax: +86-451-82589036

Received July 4, 2017; published September 4, 2017.

Supporting information for this article is available free of charge via the Internet at http://sioc-journal.cn.

Project supported by the National Natural Science Foundation of China (No. 51572052).

项目受国家自然科学基金(No. 51572052)资助. 


\section{1 引言}

燃料电池是一种新型的能源转换装置, 具有高效、 无污染等优点而被广泛关注和研究. 传统的氢氧燃料电 池是一种目前应用较为广泛的燃料电池, 但是在水下或 者太空环境中, 大量携带氧气仍然存在诸多困难, 这一 缺点大大限制了氢氧燃料电池的发展. 因此寻找一种能 够在水下和太空中大量存储和运输并且能够代替氧气 作为氧化剂的新燃料具有非常重要的研究意义. $\mathrm{H}_{2} \mathrm{O}_{2}$ 是 一种液体强还原剂, 它的还原反应是一个 2 电子过程. 与氧气的还原反应(4 电子过程)相比 ${ }^{[1,2]}$, 具有更快的动 力学和更低的活化过电势 ${ }^{[3]}$. 且液体的 $\mathrm{H}_{2} \mathrm{O}_{2}$ 储存运输方 便, 特别适合空间、水下等无氧环境; 液相进料使得以 $\mathrm{H}_{2} \mathrm{O}_{2}$ 为氧化剂的燃料电池在操作过程中不需要润湿过 程, 电池结构更简单, 体积功率密度更大, 是一类非常 有发展前景的燃料电池 ${ }^{[4]}$.

电极材料是决定燃料电池性能的重要因素之一. 目 前, 研究较多的应用于催化 $\mathrm{H}_{2} \mathrm{O}_{2}$ 电还原反应的催化剂 主要包括以下三类: (1)贵金属, 包括 $\mathrm{Au} 、 \mathrm{Pd} 、 \mathrm{Pt}$ 等以及 它们的合金 ${ }^{[8 \sim 12]}$; (2)过渡金属氧化物, 包括 $\mathrm{Co}_{3} \mathrm{O}_{4}, \mathrm{Ni}-$ $\mathrm{Co}_{2} \mathrm{O}_{4}, \mathrm{MnO}_{2}$ 等 ${ }^{[13,14]}$; (3) 贵金属和过渡金属氧化物的复 合物. $\mathrm{MnO}_{2}$ 是一种对 $\mathrm{H}_{2} \mathrm{O}_{2}$ 电还原反应具有良好的催化 性能的电极材料 ${ }^{[15 ~ 17]}$, 然而传统的 $\mathrm{MnO}_{2}$ 催化剂大多由 粉末和导电材料利用粘结剂混合制成, 粘结剂的使用不 可避免地降低了催化剂材料的利用率并使得电极的成 本增加. 因此, 研究能够直接生长在集流体表面的催化 剂材料具有非常重要的意义. 其中, 集流体的选择在电 极的设计和制备过程中起到关键作用 ${ }^{[18]}$. 泡沫镍是一 种具有三维孔道结构的导电集流体被大量应用于电极 材料的制备. 石墨烯具有巨大的比表面积和优异的电性 能是一种理想的电极材料 ${ }^{[19-21]}$. 若将石墨烯与泡沫镍 进行进一步复合, 可以制备出电性能更优异, 比表面积 更大的导电集流体.

基于以上考虑, 本文利用一步简单易操作的水热法 制备出具有良好形貌的还原氧化石墨烯修饰泡沫镍基
体, 在其表面直接生长 $\mathrm{MnO}_{2}$ 纳米片作为催化 $\mathrm{H}_{2} \mathrm{O}_{2}$ 电还 原反应的催化剂. 还原氧化石墨烯的添加可以有效增大 电极的比表面积并能起到分散 $\mathrm{MnO}_{2}$ 纳米片的作用, 避 免团聚现象的发生. 且还原氧化石墨烯和 $\mathrm{MnO}_{2}$ 纳米片 之间互相穿插, 可以为电极反应提供更多电子转移的通 道.

\section{2 结果与讨论}

\section{1 $\mathrm{MnO}_{2} / \mathrm{rGO} @ \mathrm{Ni}$ foam 电极的组成和形貌表征}

图 1(a)为 $\mathrm{MnO}_{2} / \mathrm{rGO} @ \mathrm{Ni}$ foam 电极的 XRD 谱图. 从图中可以看出该谱图上有三个较为强烈的衍射峰, 分 别位于 $44.5^{\circ}, 51.8^{\circ}$ 和 $76.3^{\circ}$, 通过与标准卡片相比对, 它 们分别对应于 $\mathrm{Ni}$ 基体(JCPDS Card NO.40-0850)的 (111)、(200)、(220)晶面, 且衍射峰比较尖锐并无其他杂 峰表明在电极的制备过程中 Ni 元素并未发生氧化或生 成其他含 $\mathrm{Ni}$ 化合物, 仍是以金属态存在. 另外, 在 $2 \theta$ 为 $26^{\circ}$ 时, 出现了一个峰宽较宽、峰强较弱的衍射峰, 对 应于 $\mathrm{rGO}$ 的衍射峰, 在 $2 \theta$ 为 $12.4^{\circ}$ 没有出现明显的 GO 衍射峰, 说明 GO 的还原程度较好. 在该 XRD 谱图上, 没有观察到明显的 $\mathrm{MnO}_{2}$ 的衍射峰, 这是由于通过水热 法制备的 $\mathrm{MnO}_{2}$ 的含量相对于基体泡沫镍而言非常少, 其衍射峰受泡沫镍衍射峰掩蔽而无法被观察到. 为了更 清楚地观察和判断是否有 $\mathrm{MnO}_{2}$ 的衍射峰存在, 将图 1(a)中绿色区域的谱线进行放大得到 1(b)图, 从 1(b)图 中可以发现, 在 $2 \theta$ 为 $37.0^{\circ}$ 和 $43.2^{\circ}$ 位置处有两个较弱的 衍射峰, 分别对应于 $\mathrm{MnO}_{2}$ (JCPDS Card NO.53-0633)的 (330)和(321)的晶面, 表明 $\mathrm{MnO}_{2}$ 被成功地制备到了 $\mathrm{rGO} @ \mathrm{Ni}$ foam 基体上. 为了进一步探究电极的组成, 我 们还对制备的 $\mathrm{rGO} @ \mathrm{Ni}$ foam 基体和 $\mathrm{MnO}_{2} / \mathrm{rGO} @ \mathrm{Ni}$ foam 电极的拉曼光谱图进行了测试和分析, 结果如支 持信息中的图 S1 所示.

图 2 分别为 $\mathrm{rGO} @ \mathrm{Ni}$ foam $(\mathrm{a}, \mathrm{c})$ 和 $\mathrm{MnO}_{2} / \mathrm{rGO} @ \mathrm{Ni}$ foam (b, d) 电极的 SEM 图片. 从图 2(a)和 2(c)中可以看 出, 经过第一步水热处理之后, $\mathrm{rGO}$ 以片状的形式均匀
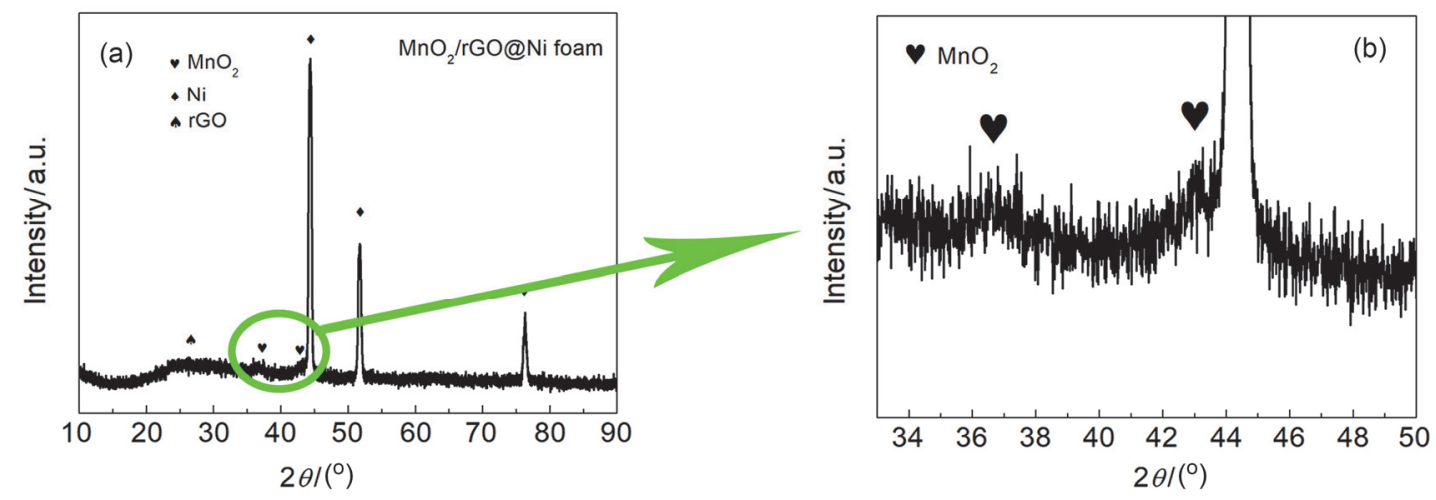

图 $1 \mathrm{MnO}_{2} / \mathrm{rGO} @ \mathrm{Ni}$ foam 电极的 XRD 谱图(a)和(a)图的局部放大图(b)

Figure 1 XRD patterns of the $\mathrm{MnO}_{2} / \mathrm{rGO} @ \mathrm{Ni}$ foam electrode (a) and partial enlargement (b) of (a) 

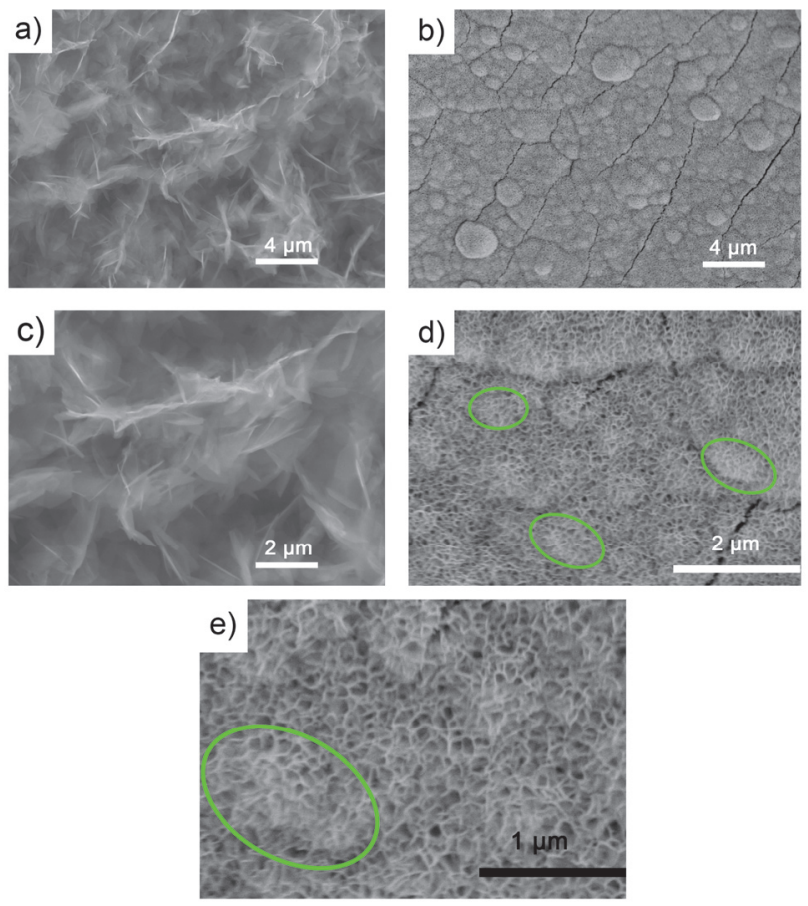

图 $2 \mathrm{rGO} @ \mathrm{Ni}$ foam 基体 $(\mathrm{a}, \mathrm{c})$ 和 $\mathrm{MnO}_{2} / \mathrm{rGO} @ \mathrm{Ni}$ foam 电极 $(\mathrm{b} \sim \mathrm{e})$ 的 SEM 图

Figure 2 SEM images of rGO@Ni foam $(\mathrm{a}, \mathrm{c})$ and $\mathrm{MnO}_{2} / \mathrm{rGO} @ \mathrm{Ni}$ foam $(b \sim e)$

地生长在泡沫镍的表面, 片与片之间互相交叉形成团簇 状结构. 从 2(c)图中可以看出, 片与片之间形成了大量 的孔道, 可以有效增大电极的比表面积, 并能使得电解 液与电极表面充分接触. 经过第二步水热处理之后, 从 图 2(b), 2(d)和 2(e)中可以看出, 在 $\mathrm{rGO}$ 片层的表面生长 了一层均匀致密的 $\mathrm{MnO}_{2}$. 从放大的 2(d)和 2(e)图中可 以看出, $\mathrm{MnO}_{2}$ 呈现出由众多的薄片组成的蜂窝状结构. 大量的孔道仍然存在在电极表面, 有利于反应物向催化 剂内部扩散并发生充分的反应. 从图 2(d)和 2(e)中绿色 区域显示, 生长的 $\mathrm{MnO}_{2}$ 层存在许多突起, 表面凹凸不 平, 这是下部的 $\mathrm{rGO}$ 的支撑作用造成的. 为了进一步探 究 $\mathrm{MnO}_{2} / \mathrm{rGO} @ \mathrm{Ni}$ foam 电极的微观形貌, 对电极进行了 透射电子显微镜的测试. 电极的 TEM 和 HRTEM 图片如 下图 3 所示. 图 3(a)中, rGO 片层厚度非常薄且带有许多 褶皱, 这些褶皱的存在可以对 $\mathrm{MnO}_{2}$ 起到很好的支撑和 分散的作用. 图 3(a)中右半部分尺寸较小的薄片为 $\mathrm{MnO}_{2}$, 宽度大约在 $50 \mathrm{~nm}$ 左右. 另外, 从图中可以看出, $\mathrm{MnO}_{2}$ 纳米片是由尺寸更小的纳米颗粒组成, 且颗粒与 颗粒之间形成了大量的纳米孔, 有利于 $\mathrm{H}_{2} \mathrm{O}_{2}$ 与 $\mathrm{MnO}_{2}$ 的充分接触和反应. 图 3(b)为图 3(a)中绿色区域的 HRTEM 图, 可以观察到明显的且有规律的晶格条纹, 宽度大约在 $0.240 \mathrm{~nm}$ 左右, 对应于 $\mathrm{MnO}_{2}$ (JCPDS Card NO.53-0633)的(330)晶面, 表明 $\mathrm{MnO}_{2}$ 被成功地制备到 了 $\mathrm{rGO} @ \mathrm{Ni}$ foam 基体上. 与此同时, 我们还对制备
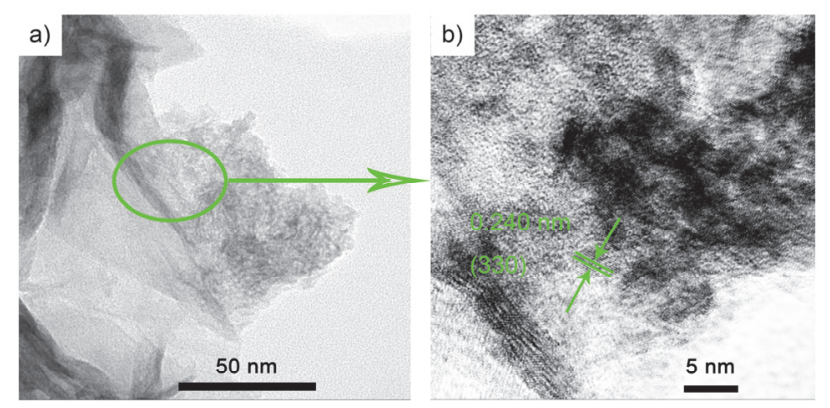

图 $3 \mathrm{MnO}_{2} / \mathrm{rGO} @ \mathrm{Ni}$ foam 电极的 TEM(a) 和 HRTEM(b)图

Figure 3 TEM (a) and HRTEM (b) of the $\mathrm{MnO}_{2} / \mathrm{rGO} @ \mathrm{Ni}$ foam electrode

的 $\mathrm{MnO}_{2} / \mathrm{rGO} @ \mathrm{Ni}$ foam 电极进行了 XPS 测试, 测试结果 如支持信息中图 S2 所示. XPS 的结果同样表明成功制备 了 $\mathrm{MnO}_{2} / \mathrm{rGO} @ \mathrm{Ni}$ foam 电极.

\section{$2.2 \mathrm{MnO}_{2} / \mathrm{rGO} @ N i$ foam 电极的电化学性能}

为了探究 $\mathrm{rGO}$ 的添加对电极催化性能的影响, 对三 种不同电极(rGO@Ni foam, $\mathrm{MnO}_{2} @ \mathrm{Ni}$ foam, $\mathrm{MnO}_{2}$ / $\mathrm{rGO} @ \mathrm{Ni}$ foam)在 $3 \mathrm{~mol} / \mathrm{L} \mathrm{NaOH}$ 和 $1.0 \mathrm{~mol} / \mathrm{L} \mathrm{H}_{2} \mathrm{O}_{2}$ 溶液 中的循环伏安曲线 $(\mathrm{CVs})$ 和计时电流曲线 $(\mathrm{CAs})$ 进行了 测试, 结果如下图 4 所示. 从图 4(a)中可以看出, rGO@ $\mathrm{Ni}$ foam 基体对 $\mathrm{H}_{2} \mathrm{O}_{2}$ 的电还原反应几乎不具有催化性 能，在测试的整个电位范围内，电流密度基本接近于 0 . 而 $\mathrm{MnO}_{2} @ \mathrm{Ni}$ foam 电极的 $\mathrm{CV}$ 曲线上的电流密度随电极 电势的负移而变大, 在电位为 $-0.8 \mathrm{~V}$ 时对应的电流密 度约为 $180 \mathrm{~mA} / \mathrm{cm}^{2}$, 这说明 $\mathrm{MnO}_{2}$ 对 $\mathrm{H}_{2} \mathrm{O}_{2}$ 的电还原反 应具有显著地催化作用. $\mathrm{MnO}_{2} / \mathrm{rGO} @ \mathrm{Ni}$ foam 电极的 $\mathrm{CV}$ 曲线与 $\mathrm{MnO}_{2} @ \mathrm{Ni}$ foam 电极的 $\mathrm{CV}$ 曲线变化趋势基 本相同, 但是在相同的电极电势下 $\mathrm{MnO}_{2} / \mathrm{rGO} @ \mathrm{Ni}$ foam 电极的 $\mathrm{CV}$ 曲线对应的电流密度更高, $-0.8 \mathrm{~V}$ 时对应的 电流密度约为 $240 \mathrm{~mA} / \mathrm{cm}^{2}$. 这表明 $\mathrm{MnO}_{2} / \mathrm{rGO} @ \mathrm{Ni}$ foam 电极的催化性能要远高于 $\mathrm{MnO}_{2} @ \mathrm{Ni}$ foam. 主要原因是 片状 $\mathrm{rGO}$ 的加入极大地提高了催化剂的比表面积, 增加 了单位面积上的电化学活性位数目, 且 $\mathrm{rGO}$ 与 $\mathrm{MnO}_{2}$ 纳 米片的互相穿插提供了更多的电子传递通道, 基于这两 点原因使其催化 $\mathrm{H}_{2} \mathrm{O}_{2}$ 电还原的速率有了显著地提升. 为了探究 $\mathrm{rGO}$ 的添加对电极比表面积的影响, 我们采用 电化学方法对 $\mathrm{MnO}_{2} / \mathrm{rGO} @ \mathrm{Ni}$ foam 电极和 $\mathrm{MnO}_{2} @ \mathrm{Ni}$ foam 电极的比表面积进行了测量, 如支持信息中图 S3 所示, 结果表明 $\mathrm{rGO}$ 的添加有效地增大了电极的比表面 积. 图 4(b)为三种电极的 CA 曲线的对比图, 显示了与 图 4(a)相同的规律. 另外从图 4(b)中可以看出, 在测试 时间范围内, $\mathrm{MnO}_{2} / \mathrm{rGO} @ \mathrm{Ni}$ foam 电极表现出良好的稳 定性, 而 $\mathrm{MnO}_{2} @ \mathrm{Ni}$ foam 电极上的电流密度随着时间的 推移呈现出明显的下降趋势, 表明 $\mathrm{rGO}$ 不仅提高了电极 的催化性能, 也使得电极的稳定性得到了改善. 

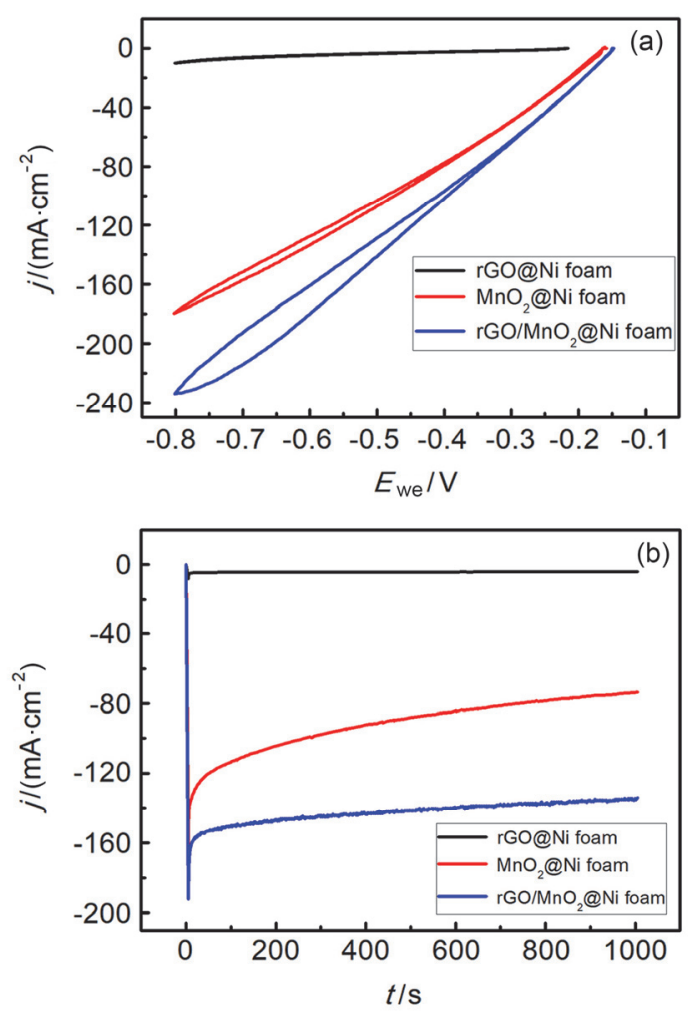

图 $4 \mathrm{rGO} @ \mathrm{Ni}$ foam, $\mathrm{MnO}_{2} @ \mathrm{Ni}$ foam 和 $\mathrm{MnO}_{2} / \mathrm{rGO} @ \mathrm{Ni}$ foam 电极在 3 $\mathrm{mol} / \mathrm{L} \mathrm{NaOH}$ 和 $1.0 \mathrm{~mol} / \mathrm{L} \mathrm{H}_{2} \mathrm{O}_{2}$ 溶液中的循环伏安(a)和计时电流(b)对 比图

Figure 4 Cyclic voltammograms (CVs) and chronoamperometric curves (CAs) of rGO@ $\mathrm{Ni}$ foam, $\mathrm{MnO}_{2} @ \mathrm{Ni}$ foam, $\mathrm{MnO}_{2} / \mathrm{rGO} @ \mathrm{Ni}$ foam electrodes in $3 \mathrm{~mol} / \mathrm{L} \mathrm{NaOH}$ and $1.0 \mathrm{~mol} / \mathrm{L} \mathrm{H}_{2} \mathrm{O}_{2}$ at a scan rate of 20 $\mathrm{mV} / \mathrm{s}$.

图 5(a)为 $\mathrm{MnO}_{2} / \mathrm{rGO} @ \mathrm{Ni}$ foam 电极在不同 $\mathrm{NaOH}$ 浓 度条件下的 $\mathrm{CV}$ 曲线对比图. 从图中可以看出, 在不同 的 $\mathrm{NaOH}$ 浓度下, 电极反应的开路电位基本保持不变, 均为 $0.15 \mathrm{~V}$ 左右. 但是电极电势达到 $-0.8 \mathrm{~V}$ 时, 不同 $\mathrm{NaOH}$ 浓度下的电解液中 $\mathrm{H}_{2} \mathrm{O}_{2}$ 的还原电流密度出现很 大差别, 分别为 $121,142,233$ 和 $176 \mathrm{~mA} / \mathrm{cm}^{2}$. 当 $\mathrm{NaOH}$ 浓度在 1 3 mol/L 范围内, 电流密度随 $\mathrm{NaOH}$ 浓度增大 而增大，但当 $\mathrm{NaOH}$ 浓度由 $3 \mathrm{~mol} / \mathrm{L}$ 增大到 $4 \mathrm{~mol} / \mathrm{L}$ 时， 电流密度随 $\mathrm{NaOH}$ 浓度增大而减小. 在此范围内, 电解 液中最佳的 $\mathrm{NaOH}$ 浓度为 $3 \mathrm{~mol} / \mathrm{L}$. 这是由于当 $\mathrm{NaOH}$ 浓度较小时, 随着 $\mathrm{NaOH}$ 浓度增大, 电解液的导电率随 之增大进而提高了反应速率. 但是当 $\mathrm{NaOH}$ 浓度提高到 一定程度时, 大量的 $\mathrm{OH}^{-}$存在于溶液中会与 $\mathrm{HO}_{2}{ }^{-}$竞争 吸附到电极表面, 减小了 $\mathrm{HO}_{2}{ }^{-}$与电极的有效接触进而 导致电极反应速率的降低, 出现电流密度随 $\mathrm{NaOH}$ 浓度 升高而下降的现象. 图 5(b)和(c)为 $\mathrm{MnO}_{2} / \mathrm{rGO} @ \mathrm{Ni}$ foam 电极在不同 $\mathrm{H}_{2} \mathrm{O}_{2}$ 浓度溶液中的 $\mathrm{CV}$ 和 $\mathrm{CA}$ 曲线, 探究了 反应物浓度对电极催化性能的影响. 从图中可以发现, 随着反应物浓度的增大, 电极上 $\mathrm{H}_{2} \mathrm{O}_{2}$ 的还原电流密度 呈现出先增大后减小的趋势. 在电位为 $-0.8 \mathrm{~V}$ 时, 电 流密度分别为 $79,113,236$ 和 $146 \mathrm{~mA} / \mathrm{cm}^{2}$. 由此可知,
最佳的 $\mathrm{H}_{2} \mathrm{O}_{2}$ 浓度为 $1.0 \mathrm{~mol} / \mathrm{L}$, 反应速率在循环伏安曲 线中的直观表现就是电流密度, 所以此时的 $\mathrm{H}_{2} \mathrm{O}_{2}$ 电还 原速率最大. 电流密度可以表示电极反应，根据法拉第 定律可推出以下方程:

$$
j=n F v=n F \frac{1}{S} \frac{\mathrm{d} c}{\mathrm{~d} t}
$$

式中 $v$ 电极反应速度 $(\mathrm{mol} / \mathrm{s}) ; S$ 电极的表面积 $\left(\mathrm{cm}^{2}\right) ; c$ 反 应物浓度 $(\mathrm{mol} / \mathrm{L}) ; t$ 反应时间 $(\mathrm{s}) ; n$ 一个反应粒子所需要 的电子数; $F$ 法拉第常数 $(\mathrm{C} / \mathrm{mol})$.

从上式中可以看出, 在电极电位不变的情况下, 电 流密度随反应物浓度的增大而增大, 即速率也随 $\mathrm{H}_{2} \mathrm{O}_{2}$ 浓度的增大而增大. 但是当过氧化氢浓度大于 1.0 $\mathrm{mol} / \mathrm{L}$ 时, 反应速率随 $\mathrm{H}_{2} \mathrm{O}_{2}$ 浓度的增大反而减小, 这是 由于 $\mathrm{H}_{2} \mathrm{O}_{2}$ 本身极易分解, $\mathrm{NaOH}$ 虽有抑制 $\mathrm{H}_{2} \mathrm{O}_{2}$ 分解的 作用, 但是当其浓度较高时, 在电解液中仍然不能稳定 存在, 会分解产生 $\mathrm{O}_{2}$. 此时, 随着 $\mathrm{H}_{2} \mathrm{O}_{2}$ 浓度增大, 分解 产生 $\mathrm{O}_{2}$ 的速率也随之增大, 产生的 $\mathrm{O}_{2}$ 不能及时逸出, 造成电极表面的气体积累, 阻隔 $\mathrm{H}_{2} \mathrm{O}_{2}$ 与催化剂的接触, $\mathrm{H}_{2} \mathrm{O}_{2}$ 直接电还原速率下降. $\mathrm{H}_{2} \mathrm{O}_{2}$ 分解是不被期望的副 反应, 不但浪费燃料, 而且在组成电池时会增加电池内 部气压, 造成电池变形, 甚至引起爆炸, 造成安全隐患. 因此选择合适的 $\mathrm{H}_{2} \mathrm{O}_{2}$ 浓度非常重要, 在本实验中的后 续电化学测试中, 都选用过氧化氢浓度为 $1.0 \mathrm{~mol} / \mathrm{L}$ 进 行测试对比分析. 从图 5(c)的 CA 曲线中可以看出, 电 极在不同 $\mathrm{H}_{2} \mathrm{O}_{2}$ 溶液中均表现出良好的稳定性, 且反映 出与 $\mathrm{CV}$ 曲线相同的规律. 当 $\mathrm{H}_{2} \mathrm{O}_{2}$ 浓度为 $1.2 \mathrm{~mol} / \mathrm{L}, \mathrm{CA}$ 曲线出现了小范围的抖动, 这是由于 $\mathrm{H}_{2} \mathrm{O}_{2}$ 分解产生的 气泡对曲线的测量产生了扰动. 图 5(d) 为电极在 3 $\mathrm{mol} / \mathrm{L} \mathrm{NaOH}$ 和 $1.0 \mathrm{~mol} / \mathrm{L} \mathrm{H}_{2} \mathrm{O}_{2}$ 溶液中不同电位条件下 的 CA 曲线, 随着电位的负移, 电流密度呈现出明显的 增大趋势. 这是由于过电位增大也就意味着反应的推动 力增大, 由 tafel 方程可知, 过电位越大, 电流密度也就 越大. 同样, 从图 5(d)中可以看出, 电极在不同电位条 件下均表现出良好的稳定性.

图6 为 $\mathrm{MnO}_{2} @ \mathrm{Ni}$ foam 和 $\mathrm{MnO}_{2} / \mathrm{rGO} @ \mathrm{Ni}$ foam 电 极在电位为 $-0.6 \mathrm{~V}$ 时在 $3 \mathrm{~mol} / \mathrm{L} \mathrm{NaOH}+X \mathrm{~mol} / \mathrm{L} \mathrm{H}_{2} \mathrm{O}_{2}$ $(X=0,1)$ 溶液中的电化学阻抗谱图. 从图中可以看出, 当不添加 $\mathrm{H}_{2} \mathrm{O}_{2}$ 时, 两种电极的电化学阻抗谱图均呈一 条直线, 表明此时电极表面没有电化学反应发生. 当加 入 $\mathrm{H}_{2} \mathrm{O}_{2}$ 后, 两种电极的电化学阻抗谱则都呈现出一个 半圆的形状, 这是由于溶液中的众多 $\mathrm{H}_{2} \mathrm{O}_{2}$ 迁移到电极 表面(固-液界面)得到两个电子被还原为 $\mathrm{OH}^{-}$, 这个半 圆的出现对应于 $\mathrm{H}_{2} \mathrm{O}_{2}$ 的电还原反应. 图 6 中的插图为两 种电极上 $\mathrm{H}_{2} \mathrm{O}_{2}$ 的电还原反应的等效电路. 由图中绿色 方框标记的区域的放大图可以看出，添加 $\mathrm{rGO}$ 后，电极 和电解液的欧姆阻抗 $(\mathrm{RS})$ 从 $1.287 \Omega$ 减小到 $0.913 \Omega$. 表 明 $\mathrm{rGO}$ 的的添加提高了电极的导电性，电极导电性的提 高为电极催化性能的提高也做出了一定贡献. 另外, 电 

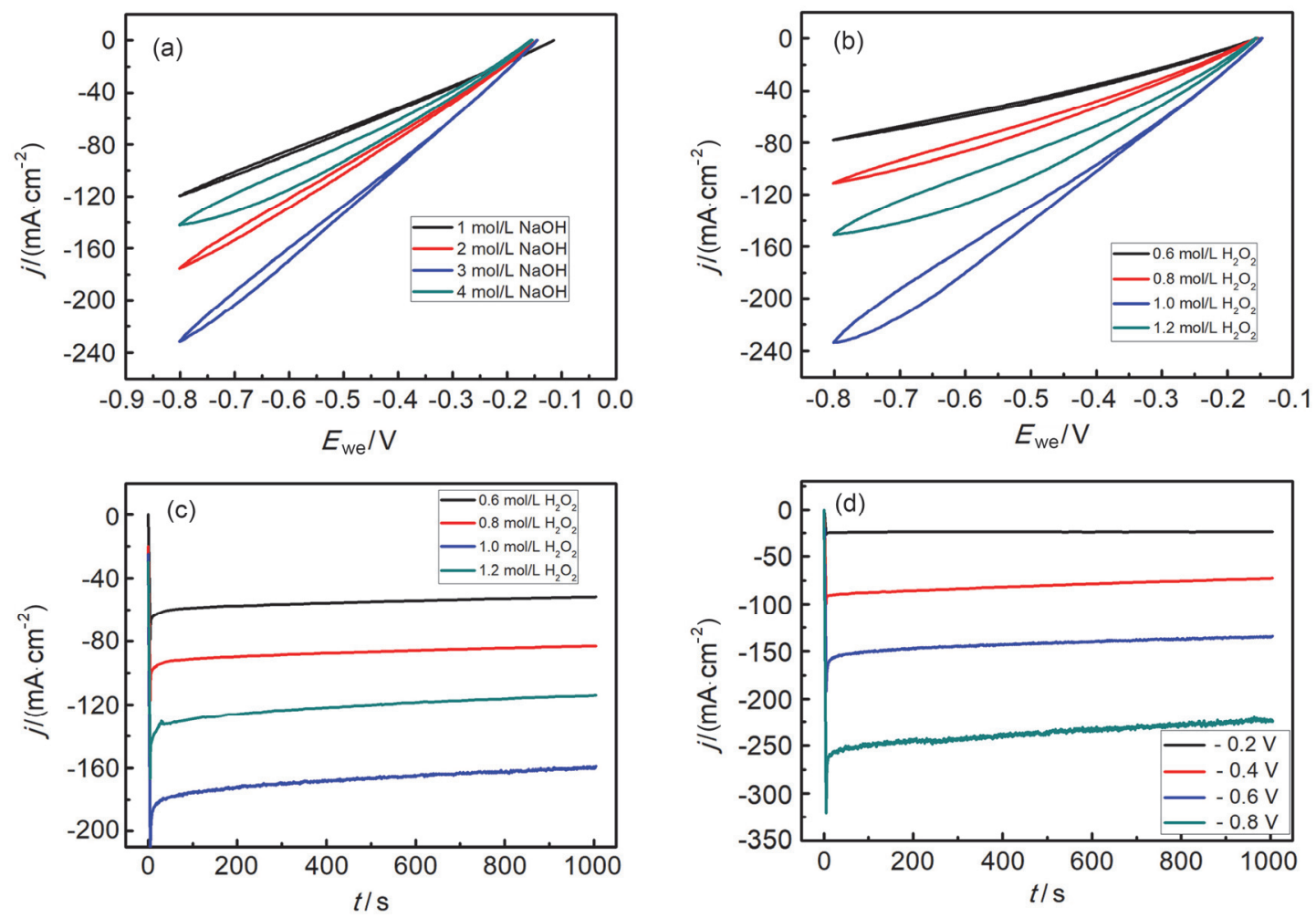

图 $5 \mathrm{MnO}_{2} / \mathrm{rGO} @ \mathrm{Ni}$ foam 电极在 $1.0 \mathrm{~mol} / \mathrm{L} \mathrm{H}_{2} \mathrm{O}_{2}$ 和 $X \mathrm{~mol} / \mathrm{L} \mathrm{NaOH}(X=1 \sim 4)$ 溶液中的循环伏安对比图(a); 在 $3.0 \mathrm{~mol} / \mathrm{L} \mathrm{NaOH}$ 和 $X \mathrm{~mol} / \mathrm{L} \mathrm{H} \mathrm{H}_{2} \mathrm{O}_{2}(X$ $=0.6,0.8,1.0,1.2$ )溶液中的循环伏安(b)和计时电流(c)对比图; 在 $1.0 \mathrm{~mol} / \mathrm{L} \mathrm{H}_{2} \mathrm{O}_{2}$ 和 $3 \mathrm{~mol} / \mathrm{L} \mathrm{NaOH}$ 溶液中不同电位时的计时电流图(d)

Figure $5 \mathrm{CVs}$ (a) of the $\mathrm{MnO}_{2} / \mathrm{rGO} @ \mathrm{Ni}$ foam electrode in solutions of different $\mathrm{NaOH}$ concentration with a fixed concentration of $\mathrm{H}_{2} \mathrm{O}_{2}(1.0$ mol/L); CVs (b) and CAs (c) of the $\mathrm{MnO}_{2} / \mathrm{rGO} @ \mathrm{Ni}$ foam electrode in $3 \mathrm{~mol} / \mathrm{L} \mathrm{NaOH}$ and different concentrations of $\mathrm{H}_{2} \mathrm{O}_{2} ; \mathrm{CAs}$ (d) of the $\mathrm{MnO}_{2} / \mathrm{rGO} @ \mathrm{Ni}$ foam electrode in $3 \mathrm{~mol} / \mathrm{L} \mathrm{NaOH}$ and $0.5 \mathrm{~mol} / \mathrm{L} \mathrm{H}_{2} \mathrm{O}_{2}$ at different applied potentials

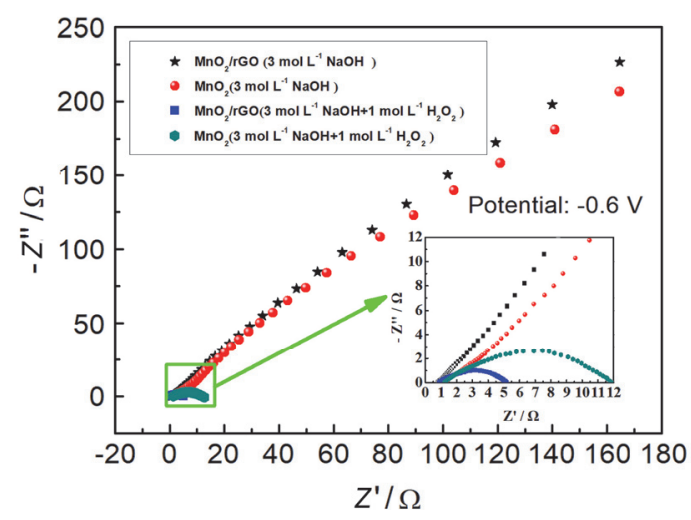

图 $6 \mathrm{MnO}_{2} @ \mathrm{Ni}$ foam 和 $\mathrm{MnO}_{2} / \mathrm{rGO} @ \mathrm{Ni}$ foam 电极在 $3 \mathrm{~mol} / \mathrm{L} \mathrm{NaOH}$ $+X \mathrm{~mol} / \mathrm{L} \mathrm{H}_{2} \mathrm{O}_{2}(X=0,1)$ 溶液中的电化学阻抗谱图. 插图为绿色方框 区域的放大图(测试电位: $-0.6 \mathrm{~V}$ )

Figure 6 Electrochemical impedance spectra of $\mathrm{MnO}_{2} @ \mathrm{Ni}$ foam and $\mathrm{MnO}_{2} / \mathrm{rGO} @ \mathrm{Ni}$ foam electrodes in solutions of $3 \mathrm{~mol} / \mathrm{L} \mathrm{NaOH}+X \mathrm{~mol} / \mathrm{L}$ $\mathrm{H}_{2} \mathrm{O}_{2}(X=0,1)$ at $-0.6 \mathrm{~V}$. The insert is the enlargement of the region marked by green rectangle

化学阻抗谱图中半圆的直径代表了电荷转移电阻, 其大 小能够反映出电极反应动力学的快慢. 从图中可以明显 地看出, 加入 $\mathrm{rGO}$ 后, 半圆的直径显著减小, 电荷转移 电阻从 $10.84 \Omega$ 减小到 $4.97 \Omega$, 表明 $\mathrm{rGO}$ 的加入加速了 电极反应的进行, 使得电极的催化性能得到了提高.
为了探究反应温度对 $\mathrm{MnO}_{2} / \mathrm{rGO} @ \mathrm{Ni}$ foam 电极催 化性能的影响, 对电极在不同温度条件下的极化曲线进 行了测量和分析, 测试的电解液仍为 $3 \mathrm{~mol} / \mathrm{L} \mathrm{NaOH}$ 和 $1.0 \mathrm{~mol} / \mathrm{L} \mathrm{H}_{2} \mathrm{O}_{2}$, 结果如下图 7 所示. 将 5 个温度 $(293$, $303,313,323,333 \mathrm{~K})$ 条件下极化曲线的塔菲尔区进行外 推, 得到与过电位为 0 的水平线的交点即可得到不同温 度条件下的交换电流密度值. 图 7(f)显示了不同温度条 件下的交换电流密度值, 可以看出, 随着温度的升高, 交换电流密度逐渐增大，分别为 $62.95,92.68,123.59$, $142.23,188.80 \mathrm{~mA} / \mathrm{cm}^{2}$, 这是由于随着温度的升高, 反 应物在体系中的传质速率增大, 进而使得交换电流密度 增大. 结合不同温度条件下的交换电流密度值和阿伦尼 乌斯公式即可求得 $\mathrm{H}_{2} \mathrm{O}_{2}$ 在 $\mathrm{MnO}_{2} / \mathrm{rGO} @ \mathrm{Ni}$ foam 电极上 直接电还原反应的活化能. 图 8 为交换电流密度的自然 对数 $\ln \left(j^{0}\right)$ 与反应温度的倒数 $(1 / T)$ 的关系曲线图. 式(2) 为阿伦尼乌斯公式:

$$
\frac{\partial \ln j}{\partial T}=\frac{E_{\mathrm{a}}}{R T^{2}}
$$

式中: $j$ 测量电流密度 $\mathrm{mA} / \mathrm{cm}^{2} ; T$ 反应温度 $(\mathrm{K}) ; E_{\mathrm{a}}$ 表观 活化能 $(\mathrm{kJ} / \mathrm{mol}) ; R$ 气体常数 $\left[8.314 \mathrm{~J} \cdot(\mathrm{mol} \cdot \mathrm{K})^{-1}\right]$.

经过计算可得, $\mathrm{H}_{2} \mathrm{O}_{2}$ 在 $\mathrm{MnO}_{2} / \mathrm{rGO} @ \mathrm{Ni}$ foam 电极上 

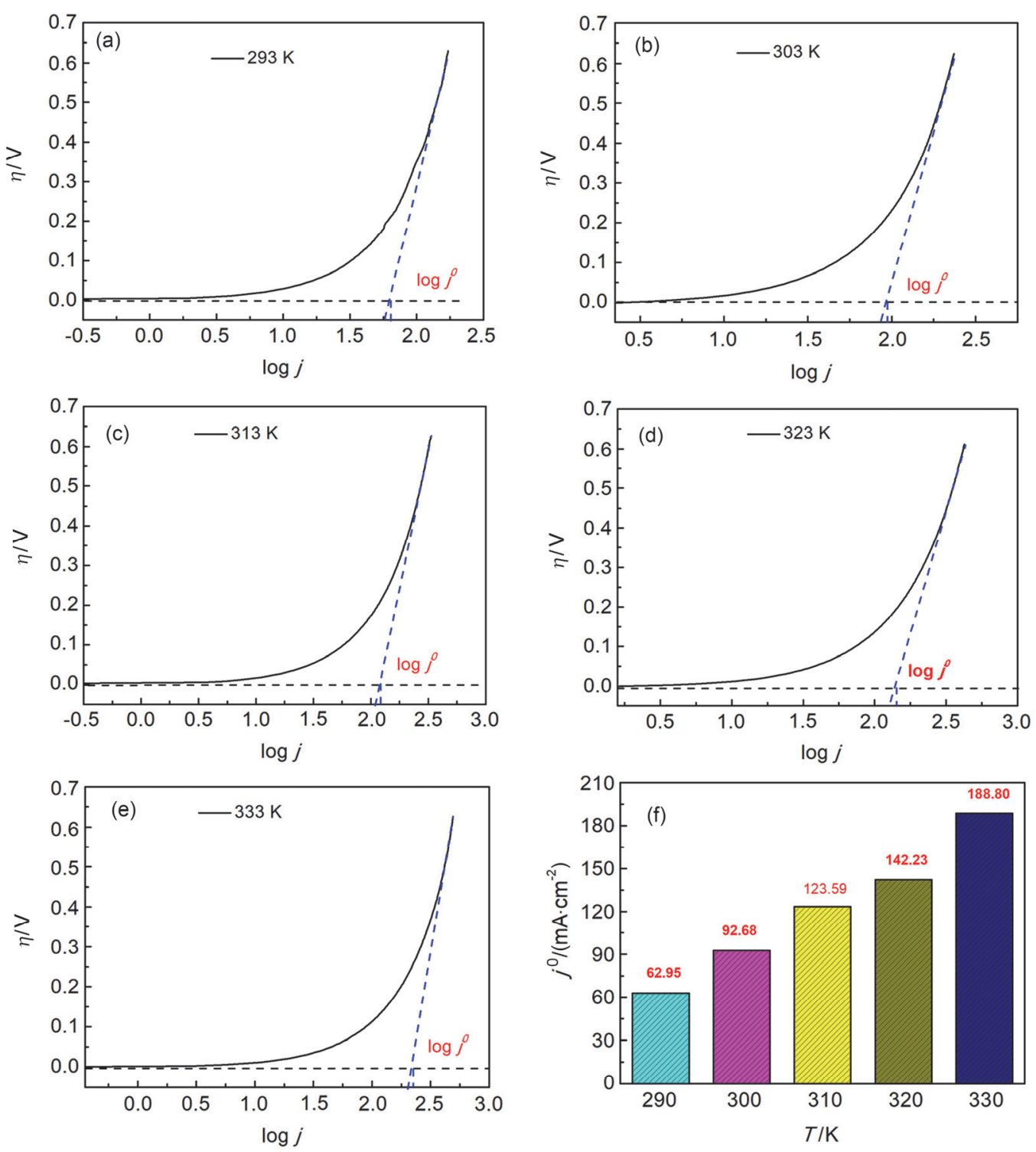

图 $7 \mathrm{MnO}_{2} / \mathrm{rGO} @ \mathrm{Ni}$ foam 电极在 $3 \mathrm{~mol} / \mathrm{L} \mathrm{NaOH}$ 和 $1.0 \mathrm{~mol} / \mathrm{L} \mathrm{H}_{2} \mathrm{O}_{2}$ 溶液中不同温度下的极化曲线(a) 293, (b) 303, (c) 313 , (d) 323 , (e) $333 \mathrm{~K}$ 和不同 温度下的交换电流密度柱状图(f)

Figure 7 Polarization curves of the $\mathrm{MnO}_{2} / \mathrm{rGO} @ \mathrm{Ni}$ foam electrode in $3 \mathrm{~mol} / \mathrm{L} \mathrm{NaOH}$ and $1.0 \mathrm{~mol} / \mathrm{L} \mathrm{H}_{2} \mathrm{O}_{2}$ at five temperatures (a) 293, (b) 303, (c) 313, (d) 323 , (e) $333 \mathrm{~K}$ and histogram (f) of exchange current densities $j^{0}\left(\mathrm{~mA} \cdot \mathrm{cm}^{-2}\right)$ under different temperatures

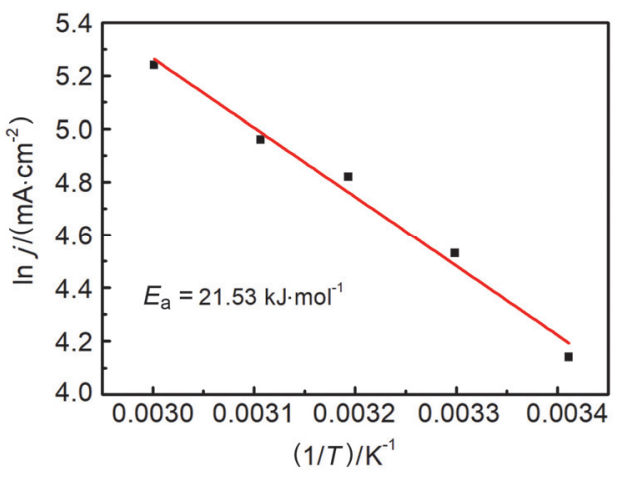

图 8 交换电流密度与温度的阿伦尼乌斯关系图

Figure 8 Arrhenius plot of the exchange current densities $j^{0}$ of $\mathrm{H}_{2} \mathrm{O}_{2}$ reduction on the $\mathrm{MnO}_{2} / \mathrm{rGO} @ \mathrm{Ni}$ foam electrode
直接电还原反应的活化能为 $21.53 \mathrm{~kJ} \cdot \mathrm{mol}^{-1}$, 明显低于 文献中报道的数值 ${ }^{[22,23]}$, 表明 $\mathrm{MnO}_{2} / \mathrm{rGO} @ \mathrm{Ni}$ foam 电极 对 $\mathrm{H}_{2} \mathrm{O}_{2}$ 的电还原反应具有优异的催化性能.

\section{3 结论}

综上所述, 本论文以 $\mathrm{rGO}$ 为添加剂, 采用一步水热 法制备了 $\mathrm{rGO} @ \mathrm{Ni}$ foam 新型导电集流体. 并通过再一 步水热法将 $\mathrm{MnO}_{2}$ 纳米片均匀生长在 $\mathrm{rGO} @ \mathrm{Ni}$ foam 基 体表面制备得到了 $\mathrm{MnO}_{2} / \mathrm{rGO} @ \mathrm{Ni}$ foam 电极. 通过各项 电化学测试结果可知, $\mathrm{MnO}_{2} / \mathrm{rGO} @ \mathrm{Ni}$ foam 电极对 $\mathrm{H}_{2} \mathrm{O}_{2}$ 的电还原反应表现出了良好的催化性能. 其中, rGO 的 添加可以增大电极的比表面积, 并能提供更多的电子转 
移通道, 使得电极的催化性能和稳定性较 $\mathrm{MnO}_{2} @ \mathrm{Ni}$ foam 电极均得到了大幅度的提升. 在 $3 \mathrm{~mol} / \mathrm{L} \mathrm{NaOH}$ 和 $1.0 \mathrm{~mol} / \mathrm{L} \mathrm{H}_{2} \mathrm{O}_{2}$ 溶液中, 电位为 $-0.8 \mathrm{~V}$ 时, $\mathrm{MnO}_{2} / \mathrm{rGO} @$ $\mathrm{Ni}$ foam 电极上 $\mathrm{H}_{2} \mathrm{O}_{2}$ 的还原电流密度可以达到 240 $\mathrm{mA} / \mathrm{cm}^{2}$, 电极反应活化能为 $21.53 \mathrm{~kJ} / \mathrm{mol}$, 充分证明制 备的 $\mathrm{MnO}_{2} / \mathrm{rGO} @ \mathrm{Ni}$ foam 是一种非常具有发展前景的 电极材料.

\section{4 实验部分}

$\mathrm{MnO}_{2} / \mathrm{rGO} @ \mathrm{Ni}$ foam 电极的制备过程如下图 9 所 示. 本实验中使用的氧化石墨烯 (GO) 是由改进的 Hummers 法制备得到 ${ }^{[24]}$. 将得到的氧化石墨烯配置成 浓度为 $1 \mathrm{mg} / \mathrm{mL}$ 的溶液待用.

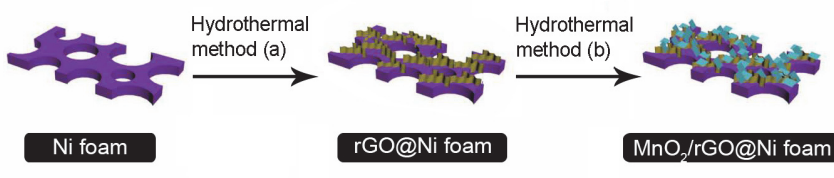

图 $9 \mathrm{MnO}_{2} / \mathrm{rGO} @ \mathrm{Ni}$ foam 电极的制备过程流程图

Figure 9 Flow diagram of the preparation of the $\mathrm{MnO}_{2} / \mathrm{rGO} @ \mathrm{Ni}$ foam electrode

首先, 对泡沫镍进行前处理. 将裁剪后的泡沫镍分 别置于丙酮和 $6 \mathrm{~mol} / \mathrm{L}$ 的盐酸溶液中超声处理 $15 \mathrm{~min}$, 然后用去离子水清洗并干燥. 量取 $15 \mathrm{~mL}$ GO 分散液稀 释至 $40 \mathrm{~mL}$, 与处理好的泡沫镍一起转移至 $100 \mathrm{~mL}$ 的 反应釜中 $160{ }^{\circ} \mathrm{C}$ 反应 $16 \mathrm{~h}$. 反应结束后, 待反应釜冷却 至室温即可得到 $\mathrm{rGO}$ 修饰泡沫镍基体(rGO@ $\mathrm{Ni}$ foam). 接着称取 $0.2528 \mathrm{~g}$ 高锰酸钾配置成 $40 \mathrm{~mL}$ 溶液, 并将其 与上一步得到的 $\mathrm{rGO} @ \mathrm{Ni}$ foam 基体一同转移至 $100 \mathrm{~mL}$ 反应釜中 $120{ }^{\circ} \mathrm{C}$ 反应 $12 \mathrm{~h}$ 即可得到 $\mathrm{MnO}_{2} / \mathrm{rGO} @ \mathrm{Ni}$ foam. 在该过程中, 反应釜内发生的反应如公式(3)所示

$$
4 \mathrm{KMnO}_{4}+3 \mathrm{C}+\mathrm{H}_{2} \mathrm{O} \rightarrow 4 \mathrm{MnO}_{2}+\mathrm{K}_{2} \mathrm{CO}_{3}+2 \mathrm{KHCO}_{3}
$$

$\mathrm{MnO}_{2} / \mathrm{rGO} @ \mathrm{Ni}$ foam 电极对 $\mathrm{H}_{2} \mathrm{O}_{2}$ 电还原反应催化 性能的测试在三电极体系中进行. 其中, 工作电极为 $\mathrm{MnO}_{2} / \mathrm{rGO} @ \mathrm{Ni}$ foam 电极, 参比电极为饱和银-氯化银
电极 $(\mathrm{Ag} / \mathrm{AgCl}, \mathrm{KCl})$, 辅助电极为碳棒. 测试的电解液 为 $\mathrm{NaOH}$ 和 $\mathrm{H}_{2} \mathrm{O}_{2}$ 的混合溶液.

\section{References}

[1] Chen, X.; Yan, H.; Xia, D. Acta Chim. Sinica 2017, 75, 189. (陈金金, 鄢慧君, 夏定国, 化学学报, 2017, 75, 189.)

[2] Li, J.; Zhang, X.; Pan, B. Chin. J. Chem. 2016, 34, 1021.

[3] Sun, L. M.; Cao, D. X.; Wang, G. L.; Lu, Y. Z.; Zhang, M. L. Acta Phys. Chim. Sin. 2008, 24, 323.

[4] Ma, J.; Choudhury, N. A.; Sahai, Y. Renew. Sust. Energ. Rev. 2010, $14,183$.

[5] Tian, Y. M.; Lei, T.; Wang, G. L.; Cao, D. X. Chem. J. Chin. Univ 2011，32，2382. (田永梅，雷婷，王贵领，曹殿学，高等学校化学 学报, 2011, 32, 2382.)

[6] Cheng, K.; Yang, F.; Yan, P.; Cao, D. X.; Yin, J. L.; Wang, G. L. Chem. J. Chin. Univ. 2014, 35, 110. (程魁, 杨帆，间鹏，曹殿学, 殷金玲, 王贵领, 高等学校化学学报, 2014, 35, 110.)

[7] Li, Z. P.; Liu, B. H.; Arai, K.; Suda, S. J. Electrochem. Soc. 2003 , $150, \mathrm{~A} 868$.

[8] Sun, L. M.; Cao, D. X.; Wang, G. L. J. Appl. Electrochem. 2008, 38, 1415.

[9] Flätgen, G.; Wasle, S.; Lübke, M.; Eickes, C.; Radhakrishnan, G.; Doblhofer, K.; Ertl, G. Electrochim. Acta 1999, 44, 4499.

[10] Gerlache, M.; Senturk, Z.; Quarin, G.; Kauffmann, J. M. Electroanal. 1997, 9, 1088

[11] Luo,Y. F.; Li, H. Z.; Chen, T. T.; Ge, C. W.; Tang, Y. W.; Chen, Y.; Lu, T. H. Electrochim. Acta 2013, 87, 839 .

[12] Yang,F.; Cheng, K.; Wu, T. H.; Zhang, Y.; Yin, J. L.; Wang, G. L.; Cao, D. X. RSC Adv. 2013, 3, 5483

[13] Wang, G. L.; Hao, S. Y.; Lu, T. H.; Cao, D. X.; Yin, C. L. Chem. J. Chin. Univ. 2010, 31，2264. (王贵领, 郝世阳，陆天虹，曹殿学, 尹翠蕾, 高等学校化学学报, 2010, 31, 2264.)

[14] Wang, G. L.; Cao, D. X.; Yin, C. L.; Gao, Y. Y.; Yin, J. L.; Cheng, L. Chem. Mater. 2009, 21, 5112 .

[15] Cheng, F.; Shen, J.; Ji, W.; Tao, Z.; Chen, J. ACS Appl. Mater. Inter. 2009, 1,460 .

[16] Ma, Y.; Wang, R.; Wang, H.; Key, J.; Ji, S. J. Power Sources 2015, 280, 526.

[17] Roche, I.; Chaînet, E.; Chatenet, M.; Vondrák, J. J. Phys. Chem. C 2007, 111, 1434

[18] Yan, P.; Zhang, D. M.; Cheng, K.; Xu, Y.; Li, Y. Y.; Ye, K.; Cao, D. X.; Wang, G. L. Chem. J. Chin. Univ. 2015, 36, 1801. (间鹏, 张栋 铭, 程魁, 徐阳, 李莹莹, 叶克, 曹殿学, 王贵领, 高等学校化学 学报, 2015, 36, 1801.)

[19] Quan, Q.; Lin, X.; Zhang, N.; Xu, Y. J. Nanoscale 2017, 9, 2398.

[20] Han, C.; Zhang, N.; Xu, Y. J. Nano Today 2016, 11, 351.

[21] Yang, M. Q.; Zhang, N.; Wang, Y.; Xu, Y. J. J. Catal. 2017, 346, 21.

[22] Hu, C.; Bai, Z.; Yang, L.; Lv, J.; Wang, K.; Guo, Y.; Cao, Y.; Zhou, J. Electrochim. Acta 2010, 55, 6036

[23] Cao, D.; Sun, L.; Wang, G.; Lv, Y.; Zhang, M. J. Electroanal. Chem. 2008, 621, 31.

[24] Marcano, D. C.; Kosynkin, D. V.; Berlin, J. M.; Sinitskii, A.; Sun, Z.; Slesarev, A.; Alemany, L. B.; Lu, W.; Tour, J. M. ACS Nano 2010, $4,4806$. 